\title{
Marktrecherche für einen Investitionsgüter-Hersteller in der Volksrepublik China
}

\author{
Hendrik Raczkowski
}

\section{Eigenvorstellung}

Nach einem zweijährigem Fachschulstudium mit der Ausbildung zum staatlich geprüften Betriebswirt mit Fachhochschulreife schrieb ich mich im September 1993 an der Technischen Fachhochschule Wildau im Studiengang Betriebswirtschaft ein. Hier begann ein Jahr später der erste Lehrgang im Fach „Chinesische Sprache und Kultur”. Das war für mich der Startschuß, über eine Laufbahn mit dem Schwerpunkt China nachzudenken. So nahm ich auch an dem ersten, vom Land Brandenburg geförderten, Sprachkurs in Schanghai teil, dem im direkten Anschluß ein 6-monatiges Praktikum bei der Siemens AG in Schanghai folgte. Fortan nahm ich verstärkt an Veranstaltungen teil, welche sich speziell mit diesem Thema beschäftigten. In diesem Zuge ergab sich auch das Angebot, im Rahmen einer Diplomarbeit für ein mittelständisches Unternehmen im Land Brandenburg eine Marktanalyse in der VR China durchzuführen. Zu diesem Zweck unternahm ich eine mehrwöchige Chinareise, die es mir ermöglichte, mit Firmen vor Ort Kontakt aufzunehmen und aktuelle Daten, die von Deutschland aus vergleichsweise schwierig zu ermitteln gewesen wären, festzustellen.

\section{Vorstellung des Unternehmens DRE/CON}

Das mittelständische Unternehmen, um das es sich bei dieser Marktanalyse handelt, ist die Firma DRE/CON Großwälzlager GmbH Eberswalde. Dieses Unternehmen stellt Großwälzlager, Seilrollen, Laufräder und Schienenzangen her. Bereits seit 1904 wurden hier von der Firma R. Adelt \& Söhne Werkzeugmaschinen produziert. 1962 wurde das Produktionsprofil der Fabrik umgewandelt und seit nunmehr 35 Jahren werden zweireihige Kugeldrehverbindungen in serienmäßiger Produktion hergestellt. Die durchschnittliche Ausbringungsmenge an Großwälzlagern beträgt ca. 4.500 Stk. pro Jahr, so sind im Laufe der Zeit ca. 95.000 Großwälzlager gefertigt und ausgeliefert worden.

Die Grundfläche des Großwälzlagerwerkes DRE/CON beträgt ca. $250.000 \mathrm{~m}^{2}$, wobei die Fläche nicht nur der Nutzung durch DRE/CON unterliegt, da sich noch 40 weitere Gewerke angesiedelt haben.

Die Firma DRE/CON Großwälzlager GmbH Eberswalde ist ein expandierendes mittelständisches Unternehmen, welches über ein Produktionsprofil auf qualitativ höchstem Niveau verfuigt. Nach mehrjähriger Konsolidierung des Unternehmens auf europäischer Ebene richtet sich nun das Augenmerk auf die wirtschaftliche Entwicklung Asiens. Spezielle Zielländer sind hierbei Indonesien, Vietnam und die VR China.

\section{Beschreibung der Aufgabenstellung}

Gerade in den verganenen Jahren wurden eine Vielzahl von Veranstaltungen wie Symposien, Messen, Konferenzen, Seminare und Foren durchgefuihrt, die alle zum Ziel hatten, deutschen Unternehmen den Weg nach Asien zu weisen. Ein Wachstumsmarkt, den die deutsche mittelständische Industrie bislang in viel zu geringem Maße bearbeitet bzw. erschlossen hat. Die VR China war hierbei ein interessantes Zielgebiet für Veranstaltungen dieser Art geworden. Das liegt nicht zuletzt darin begründet, daß in China der Aufschwung an deutlichsten in der asiatischen Region verfolgbar ist. Doch nicht nur im Rahmen dieser Veranstaltungen wird deutlich, wie wichtig dieses Thema ist. In sämtlichen Medien kann man den Werdegang Chinas mitverfolgen. Damit einher geht die Frage, wie finde ich chinesische Wirtschaftspartner? Was ist zu beachten, wo liegen die Probleme, was kostet das und welche Risiken bzw. Gefahren birgt es?

So war es ein Hauptziel meiner Diplomarbeit, sich genau mit diesen Fragen auseinander zusetzen und zu versuchen, in dem „Gewirr" und der Unzahl von Informationen eine klare Linie zu schaffen.

Die Schwerpunkte der Markanalyse lagen darin zu ermitteln,

- welche Beschaffenheit der Wälzlagermarkt in der VR China hat,

- welche Arten von Wälzlagern benötigt werden,

- welche Arten von Wälzlagern lokal hergestellt bzw. importiert werden und woher,

- wo die Stärken und die Schwächen der chinesischen Wälzlagerhersteller liegen,

- welcher Bedarf anliegt und wie dieser befriedigt werden soll,

- welcher Bedarf unter Einbeziehung von Vorhaben und Projekten größeren Umfangs existiert,

- welche Möglichkeiten eines Chinaengagements es gibt (ausschließlicher Lieferservice, Repräsentanzen vor Ort, Unternehmenszusammenschluß mit lokalen Firmen, Gründung eines eigenen Joint Ventures etc.).

\section{Herangehensweise}

Um sich mit diesem Thema umfassend beschäftigen $\mathrm{zu}$ können, ist es eingangs notwendig, sich mit der Mentalität vor Ort vertraut zu machen. Gemeint ist damit nicht nur der Umgang mit den Menschen dort, sondern auch mit den politischen, wirtschaftlichen und traditionellen Gegebenheiten. Gerade das Wissen um die Gepflogenheiten der Menschen der VR China kann sich auf 
die verschiedene Prozesse positiv auswirken. So zeichnen sich im Umgang mit den Geschäftspartnern in China deutliche Unterschiede zu denen in Deutschland oder der EU ab.

Zunächst war es wichtig, im voraus zu lokalisieren, wo entsprechende Ansprechpartner in der VR China in konzentrierter Form zu finden sind. Hierbei ergab es sich für mich, daß eine Stadt wie Schanghai dafuir der beste Ausgangspunkt ist. Hier findet man eine Vielzahl von Vertretungen der verschiedenen Arten der Wirtschaft. So galt die erste Selektion der Auswahl der Ansprechpartner. Gesucht waren dabei vor allem Unternehmen, die über ein gewisses Marktpotential und ausreichend Erfahrungen im Chinageschäft verfügen.

Zu früheren Zeiten war es ausschließlich den Ministerien vorbehalten, Kontakte mit ausländischen Partnern zu pflegen. Das hat sich heute dahingehend verändert, daß sich die Ministerien nur bei wirklich großen Projekten als Ansprechpartner anbieten und ansonsten alles weitgehend autark geregelt wird.

Da die chinesische Wirtschaft und Politik bestrebt sind, eine Öffnung und Integration zu vollziehen, wird seitens der chinesischen Regierung versucht, die ehemals staatseigenen Betriebe in eigenständige, sich selbst tragende und marktfähige Unternehmen umzuwandeln. War es früher der Fall, daß solche Betriebe keine eigenständigen ausländischen wirtschaftlichen Kontakte pflegen durften, hat es sich als günstig erwiesen, diese nach „Gesundschrumpfung“ und „Reorganisation“ selbständig auch im Außenhandel tätig werden zu lassen. Der Erfolg dieser Entwicklung läßt sich derweil an steigenden Zahlen des Bruttoinlandsproduktes und des steigenden Exports prospektieren. Kein weiteres Land der Welt hat derzeitig eine stärker wachsende Wirtschaft als die VR China und kein anderes Land zeigt eine wirtschaftliche und politische Entwicklung diesen Ausmaßes, wie es die VR China tut.

\section{Auswertung der recherchierten Daten aus Deutschland (Qualität)}

Um sicher zu gehen, daß auch alle verfügbaren Informationsquellen ausgeschöpft werden, mußten schon in Deutschland umfangreiche Recherchen durchgeführt werden. Zum einen um sicherzustellen, daß das Thema so umfassend und komplex wie nötig - jedoch auch so informativ und effizient wie möglich - bearbeitet werden kann.

So begann vorerst die Recherche in den internationalen Datenbanken des Internets. Es ist sehr wichtig, daß solche Informationsquellen nicht außer Acht gelassen werden. Gerade dieses neue Medium der Informationsbeschaffung ist in der Lage, binnen kürzester Zeit Kontakte herzustellen, um Informationen zu beschaffen, die im Normalfall einen immensen Zeit- und Geldaufwand bedeuten. Natürlich findet man solche Angaben nicht im deutschen Netz und man muß der englischen Sprache schon weitestgehend mächtig sein. Dafuir lohnt sich der Einsatz, den der Erfolg rechtfertigt. Zumal man mittels dieses Mediums innerhalb einer Stunde sowohl in Hong Kong, Kuala Lumpur, Schanghai oder Tokyo recherchiert haben kann. Bei der Internetrecherche bietet sich vor allem die Möglichkeit, aus den vielen verschiedenen Meinungen - zusammen mit den eigenen Erfahrungen - die Fragen von verschiedenen Gesichtspunkten aus zu betrachten. Allerdings sind die Netze in Asien zur Zeit noch nicht so komplex ausgebaut, wie es in Deutschland oder weiten Teilen Amerikas der Fall ist. Durch persönliche Kontakte aus China habe ich einige Verbindungen auch nach Amerika und Kanada. Von dort wurde mir ebenfalls eine umfassende Hilfe zuteil. Auch befreundete Manager bei Siemens stellten mit ihrer Meinung eine wichtige Komponente bei der Informationsbeschaffung dar.

Eine weitere Informationsquelle bildete die altbewährte Bibliothek, aus der sich, wenn auch nicht gerade die neuesten Daten, so doch zumindest Wirtschaftstrends ermitteln ließen. Als besonders beachtlich stellten sich Schriften von Consultingfirmen heraus, die trotz ihres Alters immer noch aktuell waren. Natürlich haben sich die Zahlen geändert, aber inhaltlich hatte sich nichts verschoben. Generell vertrete ich die Auffassung, daß eine Datenerhebung bzw. Marktanalyse in Form einer Projekt- oder Diplomarbeit bestenfalls der erste Schritt sein kann, um sich einen Überblick zu verschaffen und um für entscheiden zu können, ob es sinnvoll ist, in dieser Richtung weiterzudenken.

Aber sobald die Geschäftsführung entschieden hat, in eine bestimmte Richtung weiterzuarbeiten, sollte man sich an einen erfahrenen Consulter wenden. Zu den wirklich guten gehört zum Beispiel die „China Marketing Consulting”, die auch über ein Büro in Schanghai verfügt. Schließlich lassen sich 17 Jahre Chinaerfahrung und die dazugehörigen Kontakte nicht einfach anlesen!

Fachzeitschriften wie die der Bfal, Broschüren der IHK und des DIHT stellen ebenfalls wichtige Informationsquellen dar. Weiterhin gibt es zum Teil recht aussagekräftige Berichte von Banken, die man jedoch mit der nötigen Vorsicht genießen sollte, da der Aussagewert über die chinesische Wirtschaft nur schwerlich in Zahlen darstellbar ist. Ein recht beliebte Zahl, mit der des öfteren jongliert wird, ist die des Bruttoinlandprodukts (BIP). Doch wenn man von der Ausdehnung Chinas ausgeht, wird man einsehen, daß es im allgemeinen schwerlich zu schaffen sein dürfte, in einem solchen Land Zahlen für sich sprechen zu lassen. Während in den prosperierenden Küstenstädten die Wirtschaft in unerhörtem Maße boomt, kann man weit im Landesinneren noch Nomadenstämme beobachten.

Also sollte man gerade mit Daten, die man in Deutschland aus den Standardmedien erfährt, doch recht vorsichtig umgehen. Das aus noch einem weiterem Grund: Wie mir bei der Erarbeitung meiner Diplomarbeit auffiel, machen es sich viele Autoren recht leicht und schreiben einfach voneinander ab. Sogenannte „Asienexperten" schaffen es immer wieder, Bücher oder Artikel zu schreiben, obwohl es besser gewesen wäre, dies sein zu lassen. Oftmals sind die Daten veraltet, werden alte Sachverhalte mit einem neuen Datum versehen oder man kann im Extremfall zwei Bücher nebeneinander halten und ganze Artikel sind identisch.

Um auch in Deutschland an aktuelle Daten zu gelangen, kann es hilfreich sein, die aktuelle Tagespresse aufmerk- 
sam zu lesen. In Zeitungen wie „Die Welt“, „Frankfurter Allgemeine Zeitung“ und „Handelsblatt" kann man des öfteren fündig werden, wenn es um die aktuellste Zahlen bestimmter Wirtschaftsbereiche geht.

Es gibt weltweit, so auch in Deutschland, eine ganze Gruppe von sogenannten „Handelsmittlern“, die zumeist schon länger „im Geschäft“ sind und aus China stammen. Ein solcher Handelsmittler hat z. B. sein Büro in Berlin Alt-Tegel. Hier bestehen direkte Kontakte zur chinesischen Wirtschaft. Eine Zusammenarbeit kann recht fruchtbar sein. Auch kann man hier auf einen wertvollen Erfahrungsschatz zurückgreifen und nach aktuellen Geschehnissen der chinesischen Wirtschaft fragen. Solche Kontaktleute gibt es nicht nur auf chinesischer, sondern auch auf deutscher Seite. Menschen, die jahrelang in Außenstellen der deutschen Wirtschaft in China tätig waren, verfuigen häufig über wertvolle Erfahrungen im Chinageschäft und entsprechende Kontakte vor Ort. Für mich war es im Rahmen meiner Diplomarbeit möglich, durch die TFH Wildau vermittelt, sowohl deutsche als auch chinesische Kontaktpersonen kennenzulernen und von deren Wissen zu profitieren.

\section{Vor-Ort-Recherche in China (Qualität der Daten)}

Zur Qualität der Diplomarbeit kann ich sagen, daß sie durch meine Chinareise erheblich gestiegen ist. Das hängt nicht zuletzt damit zusammen, daß ich noch immer über einen gewissen Freundeskreis in Schanghai verfüge, der mich nach Kräften unterstützt hat. So war mein Praktikum in China bei der Siemens AG wieder von Nutzen, da ich mich schon während meines Praxissemesters mit einigen von ihnen gut angefreundet hatte. Das half mir, Einblicke in größere Projekte zu bekommen, die von Deutschland aus undenkbar wären. So konnte ich direkt mit verantwortlichen Managern sprechen, die an der Verwirklichung des Drei-SchluchtenStaudamms mitarbeiten. Diese haben es mir auch ermöglicht, mich auf direktem Wege mit chinesischen Managern zu treffen, wo ich im Normalfall bestenfalls seinen Stellvertreter oder einen Abteilungsleiter zu sprechen bekommen hätte.

Was für mich ebenfalls eine neue Erfahrung war, ist der Umstand, daß im Zuge der Reorganisation und des Aufbrechens alter Strukturen bzw. Seilschaften zunehmend die „alte Garde“ gegen junge dynamische Manager/innen ausgetauscht werden. So ist es mir mehr als einmal passiert, daß Verantwortlichen gegenüber saß, die jünger waren als ich (26). Ich sehe darin ein deutliches Zeichen für den neuen Geist und dem frischen Wind, der in den chinesischen Chefetagen Einzug hält.

Daß es in asiatischen Ländern Sitte ist, sich sein Personal von „Headhuntern“ rekrutieren zu lassen, war mir bereits bekannt. Doch was ich als neu empfand, waren Unternehmen, die sich ausschließlich mit der gezielten Akquirierung von Firmen jeglicher Art, Größe und Branche befassen. Diese Firmen sind darauf spezialisiert, bestimmte Zielgruppen ausländischer oder chinesischer Firmen mit Firmenprofilen, Umsatz, Mitarbeiterzahl und
Unternehmensziel ausfindig zu machen. Solche Analysen dauert je nach Auftragsumfang zwischen zwei bis vier Wochen. Ich sehe darin eine große Chance gerade für Neueinsteiger auf dem chinesischen Markt, ohne große Mühen eine bestimmte Zielgruppe zu lokalisieren. Im Durchschnitt kosten solche Erfassungen ca. 500 US-Dollar. Für detailliertere Angaben muß man jedoch mehr Geld investieren, dann kann es bis zu 1.500 USDollar oder mehr kosten. Vertreter dieser Firmen versicherten mir, daß sogar große (auch deutsche Firmen) auf dieses Potential zurückgreifen.

Eine Reise dieser Art und auch das eingebrachte Engagement lohnt sich aber erst, wenn diese Aktivitäten auch von Seiten der deutschen Mutterfirma mit voller Kraft unterstützt werden. Dann kann mit einem nennenswerten Erfolg gerechnet werden - oder das Projekt wird den Kinderschuhen nie entwachsen.

\section{Leben in China als Langnase}

Daß das Leben in China für eine „Langnase“, wie wir Europäer oder auch Amerikaner dort bezeichnet werden, nicht vergleichbar ist mit dem in unseren Breiten, braucht an dieser Stelle nicht explizit erwähnt werden. Doch möchte ich darauf hinweisen, daß so ziemlich alles, was man über China irgendwann einmal im Fernsehen gesehen oder in der Zeitung gelesen hat verglichen mit der Realität nichts ist. Als ich vor zwei Jahren das erste Mal nach Schanghai kam, erlebte ich den Kulturschock in seiner vollen Breite. Alles, was mich einmal an China so fasziniert hatte, war plötzlich gar nicht da. Schon als kleines Kind betrachtete ich das mystische und vom kulturellen Leben beherrschte China mit „glänzenden Augen“. Ich mußte nach meiner Ankunft dort feststellen, daß so ziemlich nichts davon wahr ist. Ich habe selten eine Stadt gesehen, die derartig unter ihren eigenen Umweltschäden leidet wie Schanghai. Die ersten Tage an der dortigen Universität machten mir schnell deutlich, daß $40^{\circ} \mathrm{C}$ im Schatten für einen Mitteleuropäer nicht die geeignete Umgebungstemperatur ist. Aber man kann sich mit fast jeder Situation arrangieren. Das einzige, was mir bis zum Schluß etwas verschlossen blieb, war der Zugang zum Schanghaier Essen. So kochte ich mir mein Essen regelmäßig selbst. Sicherlich ist das nicht aussagekräftig oder gleichbedeutend für die restlichen Gegenden Chinas. Doch in Schanghai fließen die Ströme an Menschen aus allen Gebieten Chinas zusammen, hier ist der Sudkessel für verarmte Bauern, glïcksuchende Geschäftemacher und den Wanderbauarbeiter, der hier sein großes Geld verdienen will. „Last but not least" sind hier auch viele weltweit operierende Firmen vertreten. Ist man als Europäer nicht bereit, diese Kultur zu respektieren, sich ihr anzupassen und zu versuchen, seinen Platz darin zu finden, sollte man recht bald den Rückflug antreten. Natürlich kann man in sogenannten „Campounds” seinen europäischen Stil bewahren und mit Gleichgesinnten hinter dicken Mauern mit Stacheldraht und einem eigenen Wachschutz in Häusern europäischer Bauart wohnen. Doch sollte einem bewußt sein, das ein solches Häuschen erst ab 4.000 US-Dollar pro Monat zu haben ist. Da meine Mit- 
tel hierfür nicht ausreichten, habe ich mitten zwischen den Chinesen gewohnt und selten so viel Freundlichkeit, Hilfsbereitschaft und Gastfreundschaft genossen.

In China zu leben, bedeutet, sich auch von den althergebrachten Vorstellungen etwas zu lösen und vom Ballast der „deutschen Zivilisation“ zu trennen. Dafür eine neue Lebensart zu erfahren und kennenzulernen, wie Menschen einer ganz anderen Kultur denken und handeln, ist meiner Meinung nach die ganzen Mühen wert.

\section{Zusammenfassung der Ergebnisse für DRE/CON und Auswertung der gewonnenen Erfahrungen in der VR China}

Abschließend kann gesagt werden, daß ein Einstieg in den chinesischen Markt für die Produktgruppen, welche DRE/CON herstellt, nicht besonders einfach ist. Dafür kam diese Überlegung schon etwas zu spät. Fakt ist aber auch, daß der Bedarf in der VR China riesig und noch lange nicht befriedigt ist. Nur mit den Geschäftskontakten ist es nicht so einfach. Schon durch den Umstand, daß China ein wahrhaft riesiges Land ist, gibt es noch reichlich zu tun. Den Weg bereiten konnte ich in der kurzen Zeit natürlich nicht, aber Wege aufzeigen. Ich denke, daß ausreichend Möglichkeiten vorhanden sind und das Marktpotential enorm ist. Doch alles, was man in diese Richtung unternimmt, soll zwar nichts übereilt, aber dennoch zügig geschehen. Denn die Konkurrenz in China ist groß. Vielleicht nicht so groß wie in Deutschland, Amerika oder Japan - aber sie wächst! Viele weltweit tätige Unternehmen haben nun schon ihren Blick Richtung Asien gelenkt oder ein entsprechendes Engagement in die strategische Planung genommen. Nun kommt es darauf an zu handeln. Daß Handlungsbedarf besteht, sieht man anhand der zahlreichen Projekte, wie z. B. der Drei-Schluchten-Staudamm oder der Hafenausbau in Schanghai. In meiner Diplomarbeit habe ich auf eine ganze Reihe weiterer Projekte und Bauvorhaben hingewiesen, mit Zahlen und auch konkreten maschinellen Anforderungen. Davon Gebrauch zu machen, kann Arbeitsplätze und Unternehmen auch hier in Deutschland auf Jahre sichern.

\section{Steht Aufwand und Nutzen im Gleichgewicht?}

Ob Aufwand und Nutzen im Gleichgewicht stehen, kann man erst nach Beginn einer Handelstätigkeit klar definieren. Die großen deutschen Firmen sind bereits seit Jahren vor Ort. Wenn sich für die Siemens AG das Chinaengagement nicht lohnen sollte, warum erhöht sie dann die Investitionen in neue Joint Venture?

Aufwand und Nutzen sind die beiden Faktoren, die gerade deutsche Unternehmer gerne schon vorab als klare Zahlen definiert habe möchten. Doch so einfach und übersichtlich geht es in China nicht. Hier kommt ein Faktor zum Tragen, der vermutlich bei der nächsten Rechtschreibreform komplett aus der deutschen Sprache entfernt wird: „Unternehmerwagnis“. Niemand als die größtenteils sozialschwache Schicht der Studenten weiß, wie sehr der Staat gerade Wenigerverdienenden mit seinen Steuern und Abgaben das Geld noch weiter aus der Tasche zieht. Ich weiß auch, daß Fördermittel und Subventionen nur solange gut sind, bis man sie braucht und die Tiefen der mißratenen Wirtschaftspolitik den deutschen Mittelstand nicht selten an den Rand der Existenz treiben. Doch ich denke, daß nur diese Unternehmerschicht flexibel und potent genug ist, ein erfolgreiches Chinaengagement durchzufuihren. Geht man in Deutschland mittlerweile von einem ROI (Return on Investment) von 5 bis 6 Jahren aus, beträgt es in China gerade mal 3 bis 4 Jahre.

Ich denke, daß eine Überlegung, in dieser Region zu investieren, keineswegs vergebens ist und man diesen Markt keineswegs aus den Augen verlieren sollte. Denn in Asien liegt die Zukunft. Das daß auch schon andere so sehen, zeigt der Ausspruch des Bundespräsidenten Roman Herzog „An Asien vorbeiplanen bedeutet an der Zukunft vorbeiplanen".

\section{Verfasser}

\section{Hendrik Raczkowski}

(4. Studienjahr, Betriebswirtschaftslehre)

Technische Fachhochschule Wildau

Bahnhofstraße

15745 Wildau 